\title{
Clinical Correlates Associated with Basic Ability of Social Life in Schizophrenia Inpatients
}

\author{
Shinichi Chiba ${ }^{*}$, Masahito Tomotake1, Masatomo Aono², Hidefumi Toshimitsuㄹ, \\ Tetsuro Ohmori ${ }^{4}$ \\ ${ }^{1}$ Department of Mental Health, Institute of Biomedical Sciences, Tokushima University Graduate School, \\ Tokushima, Japan \\ ${ }^{2}$ Department of Psychiatry, Jounan Hospital, Tokushima, Japan \\ ${ }^{3}$ Department of Psychiatry, Daiichi Hospital, Tokushima, Japan \\ ${ }^{4}$ Department of Psychiatry, Institute of Biomedical Sciences, Tokushima University Graduate School, \\ Tokushima, Japan \\ Email: "chiba.shinichi@tokushima-u.ac.jp
}

Received 13 November 2015; accepted 11 January 2016; published 14 January 2016

Copyright (C) 2016 by authors and Scientific Research Publishing Inc.

This work is licensed under the Creative Commons Attribution International License (CC BY).

http://creativecommons.org/licenses/by/4.0/

c) (†) Open Access

\begin{abstract}
Objective: The aim of the present study is to explore clinical factors associated with basic ability of social life in schizophrenia inpatients. Methods: The subjects were 50 inpatients with schizophrenia (DSM-IV). Their mean age was 53.08 ( $S D=12.08$ ) years. Social life functioning was evaluated using the Rehabilitation Evaluation of Hall and Baker (REHAB). Cognitive function was assessed with the Brief Assessment of Cognition in Schizophrenia (BACS) and clinical symptoms with the Positive and Negative Syndrome Scale (PANSS), the Calgary Depression Scale for Schizophrenia and the Drug-Induced Extrapyramidal Symptoms Scale (DIEPSS). Results: The REHAB deviant behavior score showed a significant correlation with the PANSS positive syndrome score $(r=0.55, p<$ 0.01 ) and the REHAB general behavior score had significant correlations with the PANSS positive syndrome score $(r=0.28, p<0.05)$, the PANSS negative syndrome score $(r=0.53, p<0.01)$, and the DIEPSS score $(r=0.43, p<0.01)$. However, there was no significant correlation between the scores of the REHAB and the BACS. Conclusion: These results suggest that negative and drug-induced extrapyramidal symptoms are much more important factors related to lowered basic ability of social life of schizophrenia inpatients than cognitive function.
\end{abstract}

\section{Keywords}

Schizophrenia, Inpatient, Social Life Functioning, Cognitive Function

\footnotetext{
${ }^{*}$ Corresponding author.
}

How to cite this paper: Chiba, S., Tomotake, M., Aono, M., Toshimitsu, H. and Ohmori, T. (2016) Clinical Correlates Associated with Basic Ability of Social Life in Schizophrenia Inpatients. Open Journal of Psychiatry, 6, 71-75. 


\section{Introduction}

To date, researchers have reported several clinical factors deteriorating social functioning of schizophrenia patients such as positive, negative and depressive symptoms [1]-[3]. Apart from these symptoms, recently, much more attention has been paid to cognitive dysfunction of schizophrenia patients because it might be related to lowered social and vocational activity. Previous studies have revealed [4] that cognitive dysfunction could explain $20 \%-60 \%$ of factors of social dysfunction, and dysfunction in some cognitive domains such as attention, memory and executive function has been reported to be cause of patients' poor social functioning [5]. From these findings, it seems to be clear that not only psychiatric symptoms but also cognitive function significantly influences patients' social life activity.

In the treatment of schizophrenia, early discharge from psychiatry hospital is recommended to restart patients' life in the community smoothly. For this purpose, inpatients are required to have basic ability of social life. Considering these things, evaluating inpatients' basic ability of social life seems to be crucial to see if they would be able to move from hospital to their community successfully. To assess basic ability of social life, the Rehabilitation Evaluation of Hall and Baker (REHAB) [6] has been frequently used so far, and the scale is considered as one of the most useful indices for judging the possibility of discharge from hospital.

Although basic ability of social life is an important factor in returning to the local community, there is no study investigating the factors related to lowered basic ability of social life of schizophrenia inpatients. Therefore, the purpose of the present study is to explore clinical factors associated with basic ability of social life in schizophrenia inpatients.

\section{Methods}

\subsection{Subjects}

61 Japanese inpatients with schizophrenia were recruited from three psychiatry hospitals in Japan. Data were collected between April, 2013 and March, 2015. Inclusion criterion was meeting a DSM-IV [7] diagnostic criterion for schizophrenia. The patients who were less 20 years old or had any disturbance of central nervous system including epilepsy were excluded. All subjects gave us written consent to participate in the study. As 11 of the 61 could not complete all the tests, data obtained from 50 were used for the analysis. This study was approved by the Ethics Committee of Tokushima University Hospital.

\subsection{Procedure}

We evaluated basic ability of social function, cognitive function and other clinical symptoms of the subjects by using the following measures. All assessments were done by experienced clinicians who were well trained for the use of the tools.

Level of basic ability of social life was assessed with the REHAB [6]. The REHAB is frequently used to evaluate inpatients' ability of living in the community after discharge. It is composed of two scales of "deviant behavior" and "general behavior". In this measure, higher score indicates lower level of basic ability of social life. The Japanese version of it has good reliability and validity [8].

Cognitive function was evaluated with the Brief Assessment of Cognition in Schizophrenia (BACS) [9]. The domains of cognitive function that are evaluated by the BACS are verbal memory (list learning), working memory (digit sequencing task), motor speed (token motor task), verbal fluency (category instances and controlled oral word association test), attention and speed of information processing (symbol coding), and executive function (tower of London). Higher score indicates higher level of cognitive function. The reliability and validity of the Japanese version were already confirmed [10].

Positive and negative symptoms were measured with the Positive and Negative Syndrome Scale (PANSS) [11]. It is composed of three subscales of positive syndrome, negative syndrome and general psychopathology. In the scale, higher score indicates greater level of symptom severity. The Japanese version was reported to have good reliability and validity [12].

In addition, depression symptoms were assessed with the Calgary Depression Scale for Schizophrenia (CDSS) [13]. This measure can evaluate schizophrenia patients' depressive symptoms in distinction from negative and extrapyramidal symptoms. The reliability and validity of the Japanese version were already verified [14]. Higher score indicates severer depression level. 
Drug-induced extrapyramidal symptoms were evaluated with the Drug-Induced Extrapyramidal Symptoms Scale (DIEPSS) [15]. In this study, the sum of eight individual parameters of the DIEPSS was considered severity of the drug-induced extrapyramidal symptom. Higher score means greater level of symptom severity.

\subsection{Statistical Analysis}

As several data were non-normal distribution, we used non-parametric test for correlation analysis. Spearman's rank correlation coefficients were calculated to study the relation between the REHAB scores and other clinical variables. Statistical significance was adjusted for multiple comparisons (Bonferroni correction). All statistical analyses were done with the Statistical Package for the Social Sciences, version 20.0.

\section{Results}

Demographic characteristics and means and standard deviations of the clinical indices and psychological scales are presented in Table 1. We used the chlorpromazine conversion chart [16] to determine the dosage of antipsychotic medication. All subjects were Japanese, and 30 were men and 20 women. Their mean age was 53.08 years $(\mathrm{SD}=12.08)$.

The correlations between the REHAB scores and other clinical variables are shown in Table 2. The REHAB deviant behavior score showed a significant correlation with the PANSS positive syndrome score $(r=0.55, \mathrm{p}<$ 0.01), and the REHAB general behavior score showed significant correlations with the PANSS positive syndrome score $(r=0.28, p<0.01)$, the PANSS negative syndrome score $(r=0.53, p<0.01)$ and the DIEPSS score $(\mathrm{r}=0.43, \mathrm{p}<0.01)$.

However, there was no significant correlation between the scores of the REHAB and the BACS.

\section{Discussion}

Previous studies have shown that not only clinical symptoms such as positive and negative symptoms but also cognitive dysfunction had a prominent impact on social activity and status of patients with schizophrenia [17] [18]. And it has been also reported that cognitive dysfunction has stronger impact on social functioning than positive and negative symptoms [19] [20]. Especially, lowered declarative memory and vigilance have been reported to cause poor social life [5]. In addition, dysfunction in execution function and working memory has been also reported to have negative effect on occupational and daily living function [5]. On the other hand, there are

\section{Table 1. Demographic characteristics of subjects (mean \pm SD).}

\begin{tabular}{|c|c|c|}
\hline N (Man/Woman) & & $50(30 / 20)$ \\
\hline Age (years) & & $53.08 \pm 12.08$ \\
\hline Dose of antipsychotics (mg/day) ${ }^{*}$ & & $719.90 \pm 597.27$ \\
\hline REHAB & Deviant behavior & $0.36 \pm 0.66$ \\
\hline \multirow{5}{*}{ BACS } & General behavior & $47.64 \pm 24.86$ \\
\hline & Verbal memory & $25.7 \pm 9.95$ \\
\hline & Working memory & $13.50 \pm 4.14$ \\
\hline & Motor speed & $48.12 \pm 15.84$ \\
\hline & Verbal fluency & $29.36 \pm 9.76$ \\
\hline \multirow{5}{*}{ PANSS } & Attention and speed of information processing & $32.28 \pm 11.68$ \\
\hline & Executive function & $11.24 \pm 5.62$ \\
\hline & Positive syndrome & $21.70 \pm 5.52$ \\
\hline & Negative syndrome & $22.32 \pm 5.94$ \\
\hline & General psychopathology & $44.72 \pm 8.81$ \\
\hline CDSS & & $4.08 \pm 3.77$ \\
\hline DIEPSS & & $4.70 \pm 4.33$ \\
\hline
\end{tabular}

*Chlorpromazine equivalent. REHAB, Rehabilitation Evaluation of Hall and Baker; BACS, Brief Assessment of Cognition in Schizophrenia; PANSS, Positive and Negative Syndrome Scale; CDSS, Calgary Depression Scale for Schizophrenia; DIEPSS, Drug-Induced Extrapyramidal Symptoms Scale. 
Table 2. Correlation between the REHAB and clinical indices.

\begin{tabular}{|c|c|c|c|c|c|c|c|c|c|c|}
\hline & \multicolumn{6}{|c|}{ BACS } & \multicolumn{2}{|c|}{ PANSS } & \multirow[b]{2}{*}{ CDSS } & \multirow[b]{2}{*}{ DIEPSS } \\
\hline & $\begin{array}{l}\text { Verbal } \\
\text { memory }\end{array}$ & $\begin{array}{l}\text { Working } \\
\text { memory }\end{array}$ & $\begin{array}{l}\text { Motor } \\
\text { speed }\end{array}$ & $\begin{array}{l}\text { Verbal } \\
\text { fluency }\end{array}$ & $\begin{array}{l}\text { Attention and speed } \\
\text { of information } \\
\text { processing }\end{array}$ & $\begin{array}{l}\text { Executive } \\
\text { function }\end{array}$ & $\begin{array}{l}\text { Positive } \\
\text { syndrome }\end{array}$ & $\begin{array}{l}\text { Negative } \\
\text { syndrome }\end{array}$ & & \\
\hline \multicolumn{11}{|l|}{ REHAB } \\
\hline $\begin{array}{c}\text { Deviant } \\
\text { behavior }\end{array}$ & -0.056 & 0.083 & 0.133 & -0.002 & 0.098 & 0.083 & $0.548^{* *}$ & 0.179 & -0.151 & -0.101 \\
\hline $\begin{array}{c}\text { General } \\
\text { Behavior }\end{array}$ & -0.119 & -0.039 & -0.232 & -0.109 & -0.208 & 0.049 & $0.281^{*}$ & $0.533^{* *}$ & -0.090 & $0.429^{* *}$ \\
\hline
\end{tabular}

${ }^{*} \mathrm{p}<0.05 ;{ }^{* *} \mathrm{p}<0.01$. Spearman rank correlations (Bonferroni correction). REHAB, Rehabilitation Evaluation of Hall and Baker; BACS, Brief Assessment of Cognition in Schizophrenia; PANSS, Positive and Negative Syndrome Scale; CDSS, Calgary Depression Scale for Schizophrenia; DIEPSS, Drug-Induced Extrapyramidal Symptoms Scale.

some studies reporting that cognitive dysfunction is not associated with social dysfunction [18] [21] [22].

Nowadays, early discharge from psychiatry hospital and return to the community are considered crucial from the view point of rehabilitation. Even if patients still have some psychiatric symptoms, most of them usually move from hospital to regional society when they are judged to be able to live in the society. As patients need to have enough ability of social life to return to the community, proper evaluation about it is required to predict whether they would be able to adjust to living in the community after discharge. And from these points, clinicians should focus on improving their basic ability of social life as much as possible in order to help their adjustment to the community. Therefore, it seems to be quite important to clarify the factors influencing basic ability of social life so that clinicians could know what target they should focus on in the inpatient treatment.

The present results show that cognitive function did not have any significant impact on basic social life functioning that was assessed with the REHAB. However, some clinical symptoms, especially negative and druginduced extrapyramidal symptoms were significantly related to social functioning in the study. Our results are consistent with some previous findings [18] [21] [22], and they also suggest that cognitive function might not be an important factor for basic social functioning, although it has a prominent impact on getting job and performance in work place. Nevertheless, we need to mention our subjects' characteristics. The PANSS score of the subjects was relatively high and the DIEPSS score was also high possibly because of high dose of antipsychotics. Therefore, these factors might influence the results of the present study.

As mentioned above, some of our findings seem to be not consistent with previous findings reporting cognitive dysfunction have strong influence on social functioning and status of employment [5]. The REHAB is a scale to evaluate rather basic social functioning. For example, some of the items are as follows: "How well did the patient dress himself/herself?", "How good were the patient's table manners?", "How well did the patient wash and care for himself/herself?”, "How well did the patient manage money?”. Therefore, the disagreement may come from the difference of the contents of the scales that were used.

\section{Limitations}

There are some limitations in the present study. First, as this study is a cross-sectional study, we only can guess the developmental causality. Second, our sample size is relatively small, so further research with larger sample size would be required to confirm our results. Third, since there is a possibility that there are some items overlapping to some extent in the REHAB and the PANSS, the results may have been influenced by that.

\section{Conclusion}

The current study clearly indicates that negative and drug-induced extrapyramidal symptoms are much more important factors related to lowered basic ability of social life of schizophrenia inpatients than cognitive function, suggesting that improving negative symptom and reducing extrapyramidal adverse effect might be crucial to enhance inpatients' basic functioning of social life. 


\section{Acknowledgements}

This work was supported by Japan Science and Technology Agency, CREST and by the Grants-in-Aid for Scientific Research from the Japanese Ministry of Education, Culture, Sports, Science and Technology.

\section{References}

[1] Minoru, Y., Akira, I., Shinya, I., et al. (1997) Psychiatric Symptoms and Life Disability of Patient Who Exists in Rehabilitation Ward: Using PANSS and LASMI. Japanese Bulletin of Social Psychiatry, 5, 269. (In Japanese)

[2] Tetsuya, K., Hiroyuki, O. and Toshihiko. K. (2010) Two Cases of Schizophrenia in Which Psychiatric Symptoms Are Improved and the Social Life Ability Is Improved According to the Switch to Quetiapine: Using Rehabilitation Evaluation Hall and Baker (REHAB) to Evaluate Social Life Ability. Japanese Journal of Clinical Psychopharmacology, 13, 803-822. (In Japanese)

[3] Takahumi, M., Rie, T. and Nozomi, I. (2013) Relation to Depression, Cognitive Function and Social Function of Patients with Schizophrenia. The Hokkaido Journal of Occupational Therapy, 30, 33. (In Japanese)

[4] Green, M.F., Kern, R.S., Graff, D.L., et al. (2000) Neurocognitive Deficits and Functional Outcome in Schizophrenia: Are We Measuring the "Right Stuff”? Schizophrenia Bulletin, 26, 119-136.

[5] Shinichi, N. and Masahito, F. (2004) Cognitive Function Handbook of Schizophrenia. Nankodo Co., Ltd., Tokyo.

[6] Baker, R. and Hall, J.N. (1988) REHAB: A New Assessment Instrument for Chronic Psychiatric Patients. Schizophrenia Bulletin, 14, 97-111. http://dx.doi.org/10.1093/schbul/14.1.97

[7] American Psychiatric Association (1994) Diabnostic and Statistical Manual of Mental Disorders. 4th Edition, DSM-IV, Washington DC.

[8] Toshiyuki, Y., Nobuko, H. and Akio, T. (1995) Utility of Social Life Function Used the Rehabilitation Evaluation of Hall and Baker (REHAB). Clinical Psychiatry, 37, 199-205. (In Japanese)

[9] Keefe, R.S.E., Goldberg, T.E., Harvey, P.D., et al. (2004) The Brief Assessment of Cognition in Schizophrenia: Reliability, Sensitivity, and Comparison with a Standard Neurocognitive Battery. Schizophrenia Research, 68, 283-297. http://dx.doi.org/10.1016/j.schres.2003.09.011

[10] Yasuhiro, K., Tomiki, S., Kazuyuki, N., et al. (2008) Brief Assessment of Cognition in Schizophrenia Japanese-Language Version (BACS-J). Clinical Psychiatry, 50, 913-917.

[11] Kay, S.R., Fiszbein, A. and Opler, L.A. (1987) The Positive and Negative Syndrome Scale (PANSS) for Schizophrenia. Schizophrenia Bulletin, 13, 261-276. http://dx.doi.org/10.1093/schbul/13.2.261

[12] Kay, S.R., Opler, L.A. and Fiszbein, A. (1991) Manual of the Evaluation of Psychiatric Symptoms Used the Positive and Negative Syndrome Scale (PANSS). Seiwa Shoten Co., Ltd., Tokyo.

[13] Addington, D., Addington, J. and Maticka-Tyndale, E. (1993) Assessing Depression in Schizophrenia: The Calgary Depression Scale. British Journal of Psychiatry, 163, 39-44.

[14] Yasuhiro, K., Testuro, O. and Addington, D. (2000) The Japanese Version of the Calgary Depression Scale for Schizophrenics (JCDSS). Brain and Nerve, 52, 163-166. (In Japanese)

[15] Toshiya, I. (1996) Evaluation and Diagnosis of Drug-Induced Extrapyramidal Symptoms. Seiwa Shoten Co., Ltd., Tokyo.

[16] Ataru, I. and Toshiya, I. (2006) The Chlorpromazine Conversion Chart to Determine the Dosage of Antipsychotic Medication, the Chlorpromazine Conversion Chart to Determine the Dosage of Antipsychotic Medication 2006 Versions. Japanese Journal of Clinical Psychopharmacology, 9, 1443-1447.

[17] Eack, S.M. and Newhill, C.E. (2007) Psychiatric Symptoms and Quality of Life in Schizophrenia: A Meta-Analysis. Schizophrenia Bulletin, 33, 1225-1237. http://dx.doi.org/10.1093/schbul/sbl071

[18] Rabinowitz, J., Levine, S.Z., Garibaldi, G., et al. (2012) Negative Symptoms Have Greater Impact on Functioning Than Positive Symptoms in Schizophrenia: Analysis of CATIE Date. Schizophrenia Research, 137, 147-150. http://dx.doi.org/10.1016/j.schres.2012.01.015

[19] Yasuhiro, K. and Meltzer, H.Y. (2009) Cognitive Dysfunction and Functional Outcome of Schizophrenia. Brain Science and Mental Disorders, 20, 83-88. (In Japanese)

[20] Green, M.F. (1996) What Are the Functional Consequences of Neurocognitive Deficits in Schizophrenia? American Journal of Psychiatry, 153, 321-330. http://dx.doi.org/10.1176/ajp.153.3.321

[21] Heslegrave, R.J., Awad, A.G. and Voruganti, L.N.P. (1997) The Influence of Neurocognitive Deficits and Symptoms on Quality of Life in Schizophrenia. Journal of Psychiatry \& Neuroscience, 22, 235-243.

[22] Ertugrul, A. and Ulug, B. (2002) The Influence of Neurocognitive Deficits and Symptoms on Disability in Schizophrenia. Acta Psychiatrica Scandinavica, 105, 196-201. http://dx.doi.org/10.1034/j.1600-0447.2002.1020.x 